\title{
Germanica
}

\section{Ästhetisierung der Politik: Siegfried Kracauers Totalitäre Propaganda}

Johannes VON MOLTKE : Esthétisation de la politique: Propagande totalitaire de Siegfried Kracauer

Johannes VON MOLTKE : Aestheticization of Politics: Siegfried Kracauer's

Totalitarian Propaganda

Johannes von Moltke

\section{OpenEdition \\ Journals}

Édition électronique

URL : https://journals.openedition.org/germanica/8527

DOI : 10.4000/germanica.8527

ISSN : 2107-0784

\section{Éditeur}

Université de Lille

\section{Édition imprimée}

Date de publication : 1 juin 2020

Pagination : 51-68

ISBN : 978-2-913857-45-2

ISSN : 0984-2632

Référence électronique

Johannes von Moltke, „Ästhetisierung der Politik: Siegfried Kracauers Totalitäre Propaganda“,

Germanica [Online], 66 | 2ème trimestre 2020, Online erschienen am: 02 Januar 2022, abgerufen am 06 Januar 2022. URL: http://journals.openedition.org/germanica/8527 ; DOI: https://doi.org/10.4000/ germanica.8527 


\title{
Ästhetisierung der Politik: Siegfried Kracauers Totalitäre Propaganda
}

\author{
Johannes von Moltke \\ University of Michigan
}

Siegfried Kracauers erste Arbeiten zur totalitären Propaganda standen bekanntlich unter keinem guten Stern. Das entsprechende Manuskript, 1936 vom Institut für Sozialforschung in New York in Auftrag gegeben, wurde noch während seiner Entstehung von dessen Mitgliedern kritisiert, zerpflückt und neu montiert ${ }^{1}$. Nach einigem Gerangel wurde es nie gedruckt - die verschiedenen Versionen, die zwischen Paris und New York kursierten, gelten als verschollen. Nur eine handschriftliche Fassung blieb erhalten, die 2012 als Vorlage für die Erstpublikation in der Werkausgabe diente.

Insofern könnte man von einem gescheiterten Projekt sprechen, einer Arbeit für die sprichwörtliche Schublade. Als solche hat sie jedenfalls

1. - „Ob etwas überhaupt davon brauchbar ist“, notiert Theodor W. Adorno in einem Brief an den gemeinsamen Freund Walter Benjamin, ,scheint mir noch ungewiß, fraglos dagegen, daß, wenn man überhaupt etwas zu retten unternimmt, das nur möglich sein wird, wenn man es vollständig zerschlägt und dann kleinste Bruchstücke zusammenmontiert". Theodor W. Adorno u. Walter Benjamin, Briefwechsel 1928-1940, Frankfurt a.M., Suhrkamp, 1994, S. 313. Zum Entstehungskontext siehe „Nachbemerkung und editorische Notiz“, in: Siegfried Kracauer, Werke 2.2, Studien zu Massenmedien und Propaganda, hrsg. von Christian Fleck und Bernd Stiegler, Frankfurt a.M., Suhrkamp, 2012, S. 827-849. Eine eigenständige Edition außerhalb der Kracauer Werkausgabe enthält zusätzliche Materialien (u.a. Kracauers Vorarbeiten sowie Adornos gekürzte Fassung): Siegfried Kracauer, Totalitäre Propaganda, hrsg. Bernd Stiegler unter Mitarbeit von Joachim Heck und Maren Neumann, Frankfurt a.M., Suhrkamp, 2013. 
Theodor W. Adorno eingeschätzt, der allerdings für das Scheitern zumindest mit verantwortlich war. Die Arbeit, meint er in einem Gutachten über deren ersten Teil,

ist weder von eigentlich theoretischem Wert, noch ist sie im empirischen Material zureichend fundiert, drückt aber zuweilen in literarisch höchst brauchbaren Formulierungen bestimmte Erfahrungen und Beobachtungen aus, die über die outsiderhafte Position des Autors hinaus Geltung haben ${ }^{2}$.

Das vernichtende Urteil trennt wenig subtil die gönnerhaften ,insiders" des damals in New York ansässigen Instituts für Sozialforschung vom „outsider“ Kracauer im Pariser Exil. Gleichzeitig wird dieser noch vom Bereich der Wissenschaft und „eigentlicher“" Theorie in die uneigentliche Sphäre des bloß Literarischen exkommuniziert.

Nun gibt es allerhand triftige Gründe dafür, Adorno nicht das letzte Wort zu Kracauer zu überlassen, und so möchte ich hier noch einmal nach der „Geltung“ fragen, die Adorno Kracauer herablassend und unter Vorbehalt einräumt. Der summarischen Aburteilung als theoretisch wertlos stehen nicht nur viele detaillierte, eigenständige Einsichten zu Geschichte und Begriff der totalitären Propaganda entgegen, die zunächst zu rekonstruieren sind. Darüber hinaus kommt Kracauers Propagandaarbeit ein wichtiger Stellenwert in den intertextuellen und intellektuellen Netzwerken zu, die sich während der 1930er und 1940er Jahre der Analyse von Propaganda, Demagogie, Faschismus, Totalitarismus und ,wartime communications“ widmen; hierzu gehören nicht zuletzt auch Adornos eigene Arbeiten, in denen er sich einige von Kracauers Gedanken anverwandelt. Ein weiterer Grund schließlich, warum sich die Auseinandersetzung mit Kracauers Manuskript lohnt, sind dessen kaum zu übersehende aktuelle Bezüge. Wenngleich ich diese Arbeit nicht in dem Sinne als ,Flaschenpost“ bezeichnen würde, in dem dieser Begriff immer wieder für die damaligen Arbeiten der Frankfurter Schule und deren periodische Wiederentdeckung seit Mitte der sechziger Jahre verwendet wird, so ist die verblüffende Aktualität von Kracauers Propagandaarbeit angesichts der medialen Strategien zeitgenössischer rechtspopulistischer Regime nicht von der Hand zu weisen. In dieser Hinsicht hätte eine genaue Lektüre die gewissermaßen unterirdischen Impulse zu registrieren, die auch heute noch wirksam sind.

Es geht mir im Folgenden also in erster Linie um eine intertextuelle und erschließende Lektüre, welche Kracauers Einsichten für unser Verständnis anderer Texte von Benjamins Kunstwerkaufsatz

2. - T. W. Adorno, „Gutachten über die Arbeit ,Die totalitäre Propaganda Deutschlands und Italiens', S. 1 bis S. 106, von Siegfried Kracauer", in: S. Kracauer, Werke 2.2, a.a.O., S. 821. 
bis hin zu Kracauers eigener Filmtheorie fruchtbar macht; umgekehrt erhellen diese Bezüge aber auch wesentliche Aspekte von Kracauers nur scheinbar unterbrochener Arbeit am Propagandathema. Um das zeigen zu können, gilt es zunächst, die Hauptmerkmale und zentralen Befunde der Studie Totalitäre Propaganda zu rekonstruieren.

\section{Totalitäre Propaganda: Primat des Ästhetischen}

Es handelt sich bei Kracauers Manuskript um eine facetten- und materialreiche Annäherung an das Thema - zunächst, wie es scheint, aus ideologiekritischer Sicht: Kracauer behandelt die totalitäre Propaganda (gemeint sind vor allem der italienische Faschismus und der deutsche Nationalsozialismus) auf den ersten Blick als Ideologie, welche die ohnehin schon ideologisch verfasste Wirklichkeit nochmals überwölbt, indem sie einen weiteren Keil zwischen das Verhalten von Menschen und deren materielle Interessen treibt. Propagandistische Mittel, von rhetorischen und medialen Techniken bis hin zur Ausübung von Terror, dienen dem Zweck der Verblendung und des „Massenbetrugs“, wie es später in Adornos und Horkheimers Kapitel zur Kulturindustrie heißen wird. Nicht nur, dass die faschistische Propaganda jegliches Klassenbewusstsein oder -interesse aushebelt und in der idealisierten Volksgemeinschaft aufgehen lässt; vielmehr führt totalitäre Propaganda die Menschen grundsätzlich dazu, ihr demokratisches Mitspracherecht dem totalitären Bündnis von Masse und Führerkult zu opfern. Darin entspricht also die Propaganda der Ideologie, dass sie die Macht der totalitären Herrscher idealisiert und den Beherrschten gegenüber maskiert ${ }^{3}$.

Bei der genaueren Analyse geht es Kracauer allerdings erst in zweiter Linie um ideologiekritische Demaskierung; vielmehr lenkt er die Aufmerksamkeit auch hier, wie schon einige Jahre zuvor im „Ornament der Masse“, zunächst auf die „Oberflächenäußerungen“, wie er sie dort bezeichnet. Nicht die austauschbaren Gehalte der Propaganda stehen für ihn im Vordergrund, sondern deren Erscheinungsform - ihre Maskierung4.

3. - Dieses ideologiekritische Verfahren ist mindestens teilweise pragmatisch begründet, wie die Verhandlungen mit dem IfS zeigen: Kracauer ist, wie auch an den ausführlichen und stets lobenden Horkheimer-Zitaten zu ersehen ist, bemüht, sich in die Linie des Instituts einzupassen. Dass das nicht gelingt, geht aus Adornos schroffer Ablehnung hervor: „Kracauer [gehört] weder seiner theoretischen Haltung nach verbindlich zu uns [...], noch [rangiert er] seiner Arbeitsmethode nach als wissenschaftlicher Schriftsteller überhaupt“, T.W. Adorno, „Gutachten...“, a.a.O., S. 821.

4. - ,Der Ort, den eine Epoche im Geschichtsprozeß einnimmt, ist aus der Analyse ihrer unscheinbaren Oberflächenäußerungen schlagender zu bestimmen als aus den Urteilen der Epoche über sich selbst. [...] Jene gewähren ihrer Unbewußtheit wegen einen unmittelbaren Zugang zu dem Grundgehalt des Bestehenden. An seine Erkenntnis ist umgekehrt ihre Deutung geknüpft. Der Grundgehalt einer Epoche und ihre unbeachteten Regungen erhellen sich wechselseitig.“ Siegfried Kracauer, „Das Ornament der Masse“, 
Statt stets und überall hinter der Maske des Faschismus etwa die „,ürgerlich-kapitalistische Gegenrevolution" entlarven zu wollen, fragt Kracauer:

[...] warum maskiert sich diese gerade so und nicht anders? [...] Um hierüber Aufschluss zu erhalten, darf man ersichtlich nicht gleich die Demaskierung vorwegnehmen, so als wisse man selbstverständlich schon, wer sich zu verbergen beliebt - man weiß es eben nicht [...]; man muß vielmehr aufmerksam die Maske selber betrachten. [...] Erst aus der Natur der Maske mag sich allenfalls ergeben, wie das Ungeheuer geartet ist, das sie sich aufsetzt - vorausgesetzt, daß man ihm die Maske überhaupt abreißen kann 5 .

Diese Überlegungen sind programmatisch und sie deuten methodologisch auf das voraus, was den spezifischen Charakter dieses langen Essays ausmacht: Kracauers Propagandaanalyse verfährt ästhetisch. Zwar hat die zu analysierende Maske einerseits rituelle Funktionen, die Kracauer im Zusammenhang mit dem Kultischen des Faschismus und seiner Selbstinszenierung diskutiert - sie dient, wie noch zu zeigen sein wird, der rituellen Herstellung von Massen, deren „Verflüssigung“ und Manipulation. Doch handelt es sich bei der Maske andererseits um die bildhafte Erscheinungsform dieses Kultischen (Kracauer spricht von der „Entfesselung des Massenkultes“6), also um die Oberfläche, wie sie Kracauer schon in seinen Weimarer Aufsätzen immer wieder analysiert hatte. So wie er in diesen seine Aufmerksamkeit auf die Geometrie des Massenornaments, auf „die Attribute der Fläche und der Linie“ in der „natürlichen Geometrie“ des Sozialen, auf Darstellungen städtischer Räume und auf den „Prunk der Oberfläche“ in Berliner Kinos gelenkt hatte ${ }^{7}$, so betrachtet er die Maske des Faschismus als Fläche und Form, als Oberflächenerscheinung. Folgerichtig sind auch hier die zentralen Kategorien der Analyse ästhetischer Art. Bei der „Natur der Maske“ des Faschismus geht es Kracauer um geformte Wirklichkeit und deren sinnliche Wahrnehmung. Indem er so den Akzent auf das Ästhetische setzt, knüpft Kracauer bewusst und explizit an das Selbstverständnis faschistischer Propagandisten an, die, wie zum Beispiel Hitler oder Goebbels, ausdrücklich von der Kunst der Propaganda sprechen ${ }^{8}$, und die selbst

in: Ders., Werke 5.2, Frankfurt a.M., Suhrkamp, 2011, S. 612-624, hier S. 612.

5. - S. Kracauer, „Totalitäre Propaganda“, in: Ders., Werke 2.2, a.a.O., S. 40. Zitate aus diesem Text, die im Folgenden in Klammern angegeben werden, beziehen sich auf diese Ausgabe.

6. - S. Kracauer, „Exposé: Masse und Propaganda“, in: Ders., Totalitäre Propaganda, a.a.O., S. 235.

7. - Siegfried Kracauer, „Einführung: Natürliche Geometrie“, „Knabe und Stier“ und „Kult der Zerstreuung“, in: Ders., Das Ornament der Masse, Frankfurt a.M., Suhrkamp, 1963, S. 7, S. 9 und S. 311.

8. - „Ich lernte $[\ldots]$ schon frühzeitig verstehen, daß die richtige Verwendung 
gegnerische Propaganda in „rein ästhetischer Art" würdigen (39). Ausdrücklich heißt es entsprechend schon in Kracauers Vorarbeiten, die Masse werde eben dadurch im Zuge der Propaganda ornamental, dass sie „ästhetisch reizvoll“" wirke; Propaganda vergegenwärtige die Massen sich selbst, ,in der ästhetisch verführerischen Form eines Ornamentes oder eines effektvollen Bildes"9. Indem er diesen ästhetischen Reiz ernst nimmt, begreift Kracauer seine Propagandaanalyse mithin als Aisthesis; gleichzeitig hebt er auf die Kunst der Propaganda als Formpraxis mit einer ihr eigenen Poesis $\mathrm{ab}^{10}$. Ist nämlich der Gegenstand der Propaganda zunächst die Masse, so ist ihr Verfahren ein bildendes: Propaganda bezeichnet Kracauer als „Massenbildkunst,“ die darin bestünde, ,das Ensemble der Masse so durch[zu]komponieren, dass es einen ästhetischen Reiz ausübt" (114f.) ${ }^{11}$. Die faschistischen Symbole - Fahne, fasces, Hakenkreuz, Hitlergruß - gehören ebenso zu den ästhetischen Mitteln dieser Bildkunst wie die Rhythmisierung und rhetorische Figuren der Wiederholung ${ }^{12}$. Dabei löst sich mitunter die Form von den Inhalten: Totalitäre Propaganda verfährt laut Kracauer ästhetizistisch, sie ist dem L'art pour l'art näher als dem Agitprop ${ }^{13}$. So

der Propaganda eine wirkliche Kunst darstellt“. Adolf Hitler, Mein Kampf, München, Zentralverlag der NSdAP Franz Eher Nachf., 1925, S. 193.

9. - S. Kracauer, „Vorarbeiten“, in: Ders., Totalitäre Propaganda, a.a.O., S. 253;

S. Kracauer, „Exposé: Masse und Propaganda“, a.a.O., S. 234.

10. - In dieser Hinsicht korrespondiert Kracauers Arbeit aus den 1930er Jahren etwa mit Albrecht Koschorkes Nachweis einer „Poetik des Nationalsozialismus“ in Hitlers Mein Kampf. Koschorkes Fragen an letzteres Werk sind auch die Kracauers an die faschistische Propaganda: „Welchen divergenten Lesererwartungen kommt Hitlers Werk [...] entgegen, welche neben der propagandistischen Stoßrichtung herlaufenden Inklusionsverfahren wendet er an, wen 'nimmt er mit' und wer wird nicht 'eingelassen'? Welche Reize sendet er aus, welche Art von Aufmerksamkeit wird geweckt, welche Wünsche werden befriedigt?" Albrecht Koschorke, Adolf Hitlers 'Mein Kampf': Zur Poetik des Nationalsozialismus, Berlin, Matthes \& Seitz, 2016, S. 39.

11. - Auch hier ist der Rückbezug auf das „Ornament der Masse“ deutlich, und der Hinweis auf Riefenstahl läge auf der Hand; es ist allerdings unklar, ob Kracauer Triumph des Willens zu diesem Zeitpunkt schon gesehen hatte (Gelegenheiten dazu hätte es anlässlich der Pariser Weltausstellung gegeben, wo Riefenstahl mit dem Film 1937 den „Grand Prix“ gewann).

12. - In den Vorarbeiten spricht Kracauer explizit vom „Rhythmus der Propagandawellen, seine[r] kunstvolle[n] Beschleunigung oder Verzögerung“ (S. Kracauer, „Disposition“, in: Ders., Totalitäre Propaganda, a.a.O., S. 258). Im fertigen Manuskript bezieht er sich für dieses Argument dann häufig auf den NSPädagogen und damaligen Rektor der Universität Heidelberg Ernst Krieck, wie etwa im folgenden Zitat: „Aus einem revolutionären Instinkt heraus arbeitet die nationalsozialistische Agitation vorwiegend nicht mit intellektuellen Beweisen und Argumenten sondern mit der Urkraft des Rhythmus" (103, Herv. v. mir - J.v.M); die NS Agitation, so zitiert Kracauer Krieck an anderer Stelle, arbeite „mit allem, was dem Rhythmus verwandt ist und seine erregende Kraft ausströmt" (111).

13. - „Derselbe Nihilismus, der das zynische Verhalten der Propaganda bedingt, 
folgert er: „Das Ästhetentum der Propaganda-Virtuosen wirkt sich in ihren Schöpfungen aus. Viele Maßnahmen der totalitären Propaganda sind einzig und allein darauf berechnet, ästhetisch zu faszinieren“ (74).

Wenn aber totalitäre Propaganda - so wie jede Reklame auch - mit ästhetischen Mitteln wie Symbolen, Rhythmen, Wiederholungen arbeitet, worin besteht dann die spezifische Faszination, die jene im Unterschied zu dieser bezweckt? Die Antwort auf diese Frage betrifft den aisthetischen Kern von Kracauers Analyse, läuft diese in ihrem Interesse an der Kunst der Propaganda doch letztlich auf die Frage nach deren sinnlicher Wirkung und auf die Modi sinnlicher Erkenntnis hinaus.

Propaganda bezweckt eine fundamentale und weitreichende Verunsicherung der Propagandasubjekte - und diese Verunsicherung reicht für Kracauer sehr konkret bis an die Grundfesten sinnlicher Wahrnehmung. Nicht zufällig beschreibt er die Effekte totalitärer Propaganda immer wieder in Bildern sinnlicher Desorientierung. So findet sich schon in der historischen Herleitung auf den ersten Seiten die Beschreibung der Ausgangssituation nach dem Weltkrieg als eine „Erschütterung, die zumindest vorübergehend alle Verfestigungen des psychischen und sozialen Elends aufhebt und sowohl das Individuum wie die Gesellschaft in einen labilen Zustand versetzt" (23). Diese Labilität (von lateinisch „labilis“ = leicht gleitend) macht sich die nationalsozialistische Propaganda zu Nutze: „Der Bann wirkt, man kommt ins Gleiten“"14. Indem sie sich darum bemüht, den „Rausch“ der Massen auch nach Abflauen der Kriegsbegeisterung aufrecht zu erhalten, zielt die totalitäre Propaganda auf ein permanentes "Zweifeln und Schwanken“, eine „Oszillation [elementarer] Seelenschwingungen“ (72) - kurz: auf die „Dynamisierung des starren Systems“ von Parteien und Interessen (46).

Dass und wie diese Dynamisierung nun destabilisierend auf das Subjekt wirken soll, formuliert Kracauer im Rückgriff auf zwei semantische Felder: auf optische Effekte der Spiegelung, Brechung und Blendung einerseits und auf eher haptische Dimensionen von Fließen, Fluss und Verflüssigung andererseits. Was zunächst die ersteren Konnotationen angeht, so beschreibt Kracauer den Effekt der Propaganda an zentraler Stelle als mit dem eines Spiegelkabinetts vergleichbar, welches der Totalitarismus aus ideologischen Versatzstücken regelrecht montiert. Wiederholt spricht er in dieser Hinsicht von einer Technik des „Spiegelreflexes“. Dabei bedient sich die Propaganda freizügig beim politischen Gegner, dem sie zuschreibt, „was auf's eigene Konto zu setzen wäre" (70): den Sozialdemokraten wird der Terror vorgeworfen, den die SA verübt; in einem Film wie Heimkehr begehen die Polen an

erzeugt auch den Ästhetizismus ihrer Urheber. Sie sind die modernen Vertreter des L'art-pour-l'art Prinzips [...].“ (74)

14. - S. Kracauer, „Disposition”, a.a.O., S. 251. 
Deutschen den Genozid, den die Deutschen zur gleichen Zeit an den Juden verüben ${ }^{15}$. Kaum besser lässt sich die Triftigkeit von Kracauers Analyse illustrieren als an einschlägigen Goebbels-Texten, wie etwa einem Leitartikel aus dem Januar 1941 für die NS-Zeitung Das Reich, welche schon im Titel das Verwirrspiel annonciert, das hier in antisemitischer Absicht betrieben wird: „Mimikry“ überschreibt Goebbels den Text, in dem er letztendlich den Juden ihre eigene bevorstehende Vernichtung in die Schuhe schiebt ${ }^{16}$. Indem er die Juden als unverschämte „Meister der Lüge“ hinstellt, beschreibt er sich und den nationalsozialistischen Propagandaapparat selbst; sogar noch hinter der Chuzpe, die es ebenso wie das Wort angeblich „nur unter Juden“ gebe, scheint deutlich die aggressive Tendenz der eigenen NS-Propaganda hervor. Denn als Chuzpe beschreibt Goebbels die „,bodenlose, impertinente, unglaubliche Frechheit und Unverschämtheit" des unverfrorenen Lügners. Dieser beherrsche

die Register der Wahrheitsverdrehung so genial, und er tritt dabei so sicher auf, daß er es sogar einem harmlosen Gegner gegenüber wagen kann, bei der klarsten Sache der Welt das glatte Gegenteil von dem zu sagen, was den Tatsachen entspricht. Er tut es mit einer so dreisten Frechheit, daß der Zuhörer plötzlich anfängt, unsicher zu werden ${ }^{17}$.

Unschwer erkennt man hinter den Juden - also den Opfern faschistischer Gewalt, die auf diese Weise für ihr eigenes Schicksal verantwortlich gemacht werden - die Grundzüge der Täter, also der nationalsozialistischen Propaganda, wie sie Hitler schon in Mein Kampf beschrieben hatte $^{18}$. Dort hatte er den Grundsatz vertreten, „daß in der Größe der Lüge immer ein gewisser Faktor des Geglaubtwerdens liegt"19.

Zwar könnte man denken, die Propaganda müsse sich in diesem Spiegelkabinett der eigenen Lüge überführen - Kracauer spricht hier von „Selbstenthüllungen in Spiegelschrift“ (71) und mutmaßt, es handele sich um eine Art „Bekenntniszwang! Der Drang nach Offenbarung der Wahrheit tritt als Spiegelreflex hervor"20. Diese Selbstenthüllungen sind aber nur lesbar aus der analytischen Distanz - was auch heißt: aus der Distanz des Exils, aus der Kracauer Hitlers und Goebbels' Schriften in der Bibliothèque Nationale studiert oder aus welcher Horkheimer

15. - Vgl. Johannes von Moltke, The Curious Humanist: Siegfried Kracauer in America, Berkeley, University of California Press, 2015, S. 63-79.

16. - Joseph Goebbels, „Mimikry“, Das Reich, 20. Juli 1941.

17. - Ebd.

18. - Goebbels lehnt sich in einigen Formulierungen seiner antisemitischen Hetze direkt an Passagen aus Mein Kampf an. Siehe dort S. 66f., 253, etc.

19. - Ebd., 252.

20. - S. Kracauer, „Vorarbeiten“, a.a.O., S. 250. 
und Adorno wenig später die pathische Projektion als Kern antisemitischer Propaganda bestimmen und Adorno schreiben kann, faschistische Demagogen wie der Radiopastor Martin Luther Thomas „blame the prospective victim for the very same crime one wants to commit oneself" 21 . Indes gewährleistet der totalitäre Zusammenhang und die schiere Größe der Lüge den Fortbestand des Regimes, und entzieht den Subjekten jeglichen Boden und Erfahrungshorizont, von dem aus die Spiegelschrift sich entschlüsseln ließe. Kracauer beschreibt mithin den Effekt der Propaganda als eine abgrundtiefe Desorientierung des Subjekts, das aus dem Spiegelkabinett keinen Ausweg weiß.

Stattdessen gerät es durch den Schulterschluss von Terror und Propaganda unter ,hydraulischen Druck“. Wie ein groß angelegtes Ingenieursprojekt, das sich der Massen als Baumaterial bedient, verflüssigt die Propaganda „den festen Komplex, zu dem der ideelle Oberbau und seine Fundamente zusammengeschweißt sind" und leitet dadurch statische gesellschaftliche Verhältnisse in dynamische über (46). Denn als Massenbewegung setze der Nationalsozialismus, so einer seiner Apologeten, „die Kunst der Massenerregung [voraus]: Masse muss flüssig werden, wenn sie gestaltet sein soll“" (ebd.). Das Subjekt, das seine Individualität schon längst an die Masse hat abgeben müssen, wird buchstäblich gänzlich liquidiert: es wird flüssig gemacht und zergeht.

Diese beiden Beschreibungsansätze von Propaganda als Verspiegelung und Verflüssigung haben den Verweis auf die grundsätzliche Desorientierung des Subjekts gemeinsam. Obwohl Kracauer auch diese Desorientierung zunächst auf der Ebene der Sinneswahrnehmung als „Schwindelgefühl“" beschreibt, das die Massen zwinge, die Augen zu schließen und die Frage nach der Richtigkeit irgendeiner propagandistischen These zurückzustellen" (72), geht es dabei letztlich um nichts Geringeres als die „Veränderung der psycho-physischen Struktur der Menschen“"(49)22.

Nun könnte man meinen, diese ästhetische Analyse der Propaganda vernachlässige deren eigentliche Dimension, also das Politische. So ungefähr dürfte der Tenor der Diskussionen in New York gewesen sein, wo Horkheimer und Adorno sich weigerten, dem „Pseudohumanismus“ der Frankfurter Zeitung, der sie Kracauer auch lange nach dessen Rausschmiss noch zurechneten, irgendwelche Konzessionen zu machen,

21. - Zum Antisemitismus s. Theodor W. Adorno/Max Horkheimer, „Elemente des Antisemitismus“, in: Dies., Dialektik der Aufklärung, Frankfurt a.M., Fischer, 1988, S. 177217; zu Martin Luther Thomas, s. Theodor W. Adorno, The Psychological Technique of Martin Luther Thomas' Radio Addresses, Stanford, Stanford University Press, 2000, S. 12.

22. - Die Propaganda muss ,die psycho-physische Struktur der Menschen verändern [...], um den jeweils von ihr angepriesenen Attrappen einige Anziehungskraft verleihen zu können.“ (90) 
und eine mehr auf ökonomische Zusammenhänge grundierende materialistische Analyse einforderten ${ }^{23}$. Und in der Tat verfährt Kracauer in gewisser Weise weniger materialistisch als idealistisch, wenn er von Propaganda als ,ideeller Montage“ „,freigesetzter“ Ideen spricht, die losgelöst von irgendwelchen Interessen und nur im Dienst eines nihilistischen Machtwillens manipuliert werden (vgl. 45). Statt allerdings den mangelnden Materialismus einer solchen Analyse einzuklagen ${ }^{24}$, halte ich es für sinnvoller, den Formen nachzugehen, in denen diese ästhetische Betrachtung der Propaganda produktiv wird - in Kracauers eigenen Schriften wie auch in denen seiner Weg- und Zeitgenossen. Wie zu zeigen sein wird, fügt sich in dieser intertextuellen Perspektive notwendigerweise auch die Politik zu Kracauers Propagandaästhetik.

\section{Propaganda, Kunstwerk, Film: Zur Dialektik von Ästhetik und Politik}

Kracauer arbeitete im Pariser Exil ab Mitte 1937 ungefähr ein Jahr lang an dem Propagandamanuskript, das er zwischen Oktober desselben Jahres und Juni 1938 kapitelweise an das Institut für Sozialforschung in New York übermittelte. Wie üblich exzerpierte er für die Arbeit zunächst die für ihn wichtige Literatur auf einzelnen Karteikarten, was ihn zweifellos regelmäßig in die Bibliothèque Nationale geführt haben wird; dort wird er auch immer wieder seinem Freund Walter Benjamin begegnet sein, der zu dieser Zeit für das avisierte Passagenwerk an einem Baudelaire-Aufsatz saß. Doch verbindet noch weit mehr die beiden im Pariser Exil angefertigten Arbeiten. Denn Benjamin hatte erst im Vorjahr eine Auftragsarbeit für das Institut für Sozialforschung an dessen Zeitschrift abgeschickt, wo sie 1936 auf Französisch unter dem Titel „L'œuvre d'art à l'époque de sa reproduction mécanisée“ erschienen war ${ }^{25}$. Seine „Reproduktionsarbeit“, wie Benjamin einen seiner wohl berühmtesten Texte nannte, sollte ihn noch bis zu seinem Tod 1940 beschäftigen. Benjamin fertigte auch nach dem Erscheinen in der Zeitschrift für Sozialforschung und in der Hoffnung auf weitere

23. — Vgl. Adorno an Horkheimer, 22. September 1937: ,„[...] hatte auch die größten Bedenken angemeldet und ihn auf die ökonomischen Zusammenhänge - auch die mit der Reklame - verwiesen. Es ist aber offenbar sehr schwer ihn zu lenken, wenn man sich nicht wirklich Zeit dazu nimmt.“ Theodor W. Adorno, Briefe und Briefwechsel IV.1, Frankfurt a.M., Suhrkamp, 2003, S. 411.

24. - In Form detaillierter Herleitungen, wenn nicht aus ökonomischen, so doch aus konkreten historischen Zusammenhängen nach dem Ersten Weltkrieg gehören materialistische Überlegungen durchaus zu Kracauers Analyse.

25. - Wieder abgedruckt in Walter Benjamin, Das Kunstwerk im Zeitalter seiner technischen Reproduzierbarkeit, Werke und Nachlaß: Kritische Gesamtausgabe Band 16, Frankfurt a.M., Suhrkamp, 2012, S. 164-199. 
Veröffentlichungen der Arbeit auf Englisch und in der deutschsprachigen Exilpresse Notizen an, und auch in seinen Briefen kehrt er immer wieder zu den Themen der „Reproduktionsarbeit" zurück. Von deren verschiedenen Versionen hat auch Kracauer während seiner Arbeit an Totalitäre Propaganda eine einsehen dürfen ${ }^{26}$. In seinem Abschnitt über „Propaganda als Instrument der Macht" bezieht er sich ausdrücklich auf „einen noch unveröffentlichten Teil seiner [Benjamins] Abhandlung", der - selbst wiederum in Anspielung auf Kracauers Weimarer Aufsätze - die Konstitution der Masse zum Gegenstand hat. Wie Philippe Despoix und Nia Perivolaropoulou gezeigt haben ${ }^{27}$, liegt im Begriff der Masse - als Kult, als Ornament, und als soziale Kraft - in der Tat ein wichtiger Strang der mehr oder weniger explizit ausgetragenen Debatte zwischen Kracauer und Benjamin, die sich bis in die zwanziger Jahre zurück verfolgen lässt. Doch lässt sich die gegenseitige intertextuelle Relevanz der beiden Essays, die Mitte der dreißiger Jahre entstehen - also der „Reproduktionsarbeit“ einerseits und der Totalitären Propaganda andererseits - noch auf andere Weise verorten. Denn die Lektüre von Kracauers Schrift kann selbst noch einige der meistzitierten Passagen und Dimensionen von Benjamins Kunstwerk-Aufsatz erhellen. Umgekehrt zeigt der Rückbezug auf Benjamin auch an Kracauers Essay wichtige Details auf, die dann im weiteren Verlauf von dessen Arbeiten zum Film relevant werden.

So fällt auf, dass Benjamin und Kracauer in den gleichen Kategorien denken: für den Kunstwerkaufsatz des einen wie für die Propagandaarbeit des anderen gleichermaßen bedeutend sind zentrale Begriffe wie Masse, Montage, Schock - und mithin die Frage des Ästhetischen in der Moderne. Für Kracauer wie für Benjamin geht es dabei letztlich um die Möglichkeit bzw. den Verlust von Erfahrung. Während Benjamin nachzeichnet, inwiefern die Reproduktionstechnologie tief in die Konstitution nicht nur des Wahrnehmungsapparats, sondern von Subjektivität selbst eingreift und den Erfahrungshorizont zumal im Kino in eine Montage schockhafter Erlebnisse überführt, notiert Kracauer, wie oben gezeigt, wie die nationalsozialistische Propaganda das Subjekt ebenfalls durch Montagen und Schocks unter Druck setzt, verflüssigt und damit im Grunde erfahrungsunfähig macht. Nun ist dieser Prozess bei Benjamin bekanntlich ambivalent gedacht: Verleihen andere seiner Essays, etwa zum Erzähler, dem Erfahrungsverlust nostalgische Konturen, so erkennt Benjamin im Kunstwerk-Essay in der

26. - Hierbei dürfte es sich der Editionsgeschichte nach zu schließen um die in der kritischen Gesamtausgabe als fünfte Version bezeichnete handeln; im Folgenden zitiere ich deshalb aus dieser Fassung, in: ebd., S. 207-250.

27. - Vgl. hierzu die Artikel von Philippe Despoix und Nia Perivolaropoulou in diesem Heft. 
Hinwendung zu Schock, Fragmentierung und Montage durchaus ein emanzipatorisches Potenzial. Dennoch sieht er dieses, wie insbesondere am Anfang und Ende des Aufsatzes deutlich wird, stets durch die faschistische Vereinnahmung bedroht, die noch den Krieg zum ästhetischen Ereignis macht.

Kracauers ästhetische Analyse der faschistischen Propaganda setzt aber genau dort an, wo Benjamin aufhört: nämlich bei der Ästhetisierung der Politik. Es entsteht bei genauerem Hinsehen der Eindruck, als führe Kracauer in seiner Arbeit den Nachweis der berühmten abschließenden These, die Benjamin in seinem Kunstwerkabsatz mit Bezug auf Marinetti anreißt, ohne sie im Detail zu belegen. Dort behauptet er, der Faschismus suche den Massen „Ausdruck in [der] Konservierung [der Eigentumsverhältnisse] zu geben": darin, dass diese Verhältnisse ebenso wie die Massen aber durch den Faschismus unverändert bleiben, besteht für Benjamin die „Ästhetisierung des politischen Lebens“, welche nur im Krieg enden könne. Darauf habe der Kommunismus mit der „Politisierung der Kunst“" zu antworten ${ }^{28}$.

Vollendet sich auf diese Weise bei Benjamin das L'art pour l'art im Krieg, so sieht es Kracauer ausdrücklich in der Propaganda verwirklicht. Weitaus skeptischer als jener verfolgt Kracauer die Ästhetisierung der Politik in die Windungen einer als Kunst aufgefassten Propaganda und deren Wirkungen. Er beschreibt an der Propaganda, wie beliebig sie mit ihrem Material verfährt. Da die Propaganda zum Selbstzweck wird (Adorno formuliert wenig später ähnlich, ,propaganda is the ultimate content of [...] propaganda" 29 ) sind auch ihre Strategien der Ästhetisierung für Kracauer in gewisser Hinsicht formalistisch: „Man organisiert in beliebigem Material Schocks, um einem Nachlassen der Spannung zu begegnen“ (168). Anders als Benjamin siedelt also Kracauer die Effektivität des Schocks auf Seiten der Propaganda und des Faschismus an, statt auf der Seite einer entstehenden Resistenz, einer „Befreiung“ der Schockwirkung des Films aus der moralischen Emballage, wie Benjamin sie nachzeichnet ${ }^{30}$.

Umgekehrt lässt sich auch feststellen, dass der Bezug zu Benjamin Kracauers eigene, weitere Arbeit am Propagandathema erhellt. Mehr noch: der intertextuelle Bezug erlaubt es aufzuzeigen, wie diese Arbeit sich letztlich auf subtile und überraschende Weise auf Kracauers Filmtheorie als solche auswirkt. Als er sich nämlich in seiner ersten Arbeit für das MoMA in den USA 1941 wieder dem Thema widmet,

28. - W. Benjamin, „Das Kunstwerk im Zeitalter seiner technischen Reproduzierbarkeit”, 5. Fassung, in: Ders., Das Kunstwerk..., a.a.O., S. 248 u. 250.

29. - T.W. Adorno, The Psychological Technique..., a.a.O., S. 32.

30. — W. Benjamin, „Das Kunstwerk...“, a.a.O., S. 244. 
lassen sich zwei wesentliche Verschiebungen feststellen. In der 1941 entstandenen Studie „Propaganda and the Nazi War Film“ wird nun zum einen der Film zentral - was pragmatische Hintergründe hat, aber auch systematisch wesentlich ist. Es geht Kracauer hier um langformatige Propagandastreifen wie Sieg im Westen und Feldzug in Polen; in den USA sieht er vermutlich auch zum ersten Mal Riefenstahls Triumph des Willens und analysiert diesen ebenso wie die vom Heer produzierten Wochenschauen nun mit genau jenen Begriffen, die er fünf Jahre zuvor in Paris entwickelt hatte. Auch hier geht es um den Angriff der Bilder auf die Konstitution des Zuschauers, um Schock und Überwältigung, um die psycho-physische Struktur des Subjekts ${ }^{31}$.

Doch ergibt sich nun im Rückbezug auf Benjamin noch eine zweite Überblendung, die ich in der These zusammenfassen möchte: Kracauer überlagert den Benjamin'schen Chiasmus von Ästhetik und Politik mit dem von Film und Wirklichkeit. Wo Benjamin davor gewarnt hatte, der Faschismus ästhetisiere die Politik, stellt Kracauer nun fest: in der faschistischen Propaganda überwölbt der Film die Wirklichkeit mit allen ihm zur Verfügung stehenden ästhetischen Mitteln, von elliptischer Montage bis zur unablässigen Bewegung, welche Kracauer wieder, ganz wie im früheren Text, auf ihre Zuschauereffekte hin analysiert. So findet sich auch hier die Diagnose einer tiefgreifenden Verunsicherung, wobei das Bild vom Spiegelkabinett nun abgelöst wird von einem noch weiter gehenden Realitätsverlust, den das „oszillierende“ Subjekt im Propagandafilm erleidet: dieser ,attempts to maintain in the spectator oscillations that, if they really could be maintained, would make him indifferent to truth or untruth and alienate him from reality forever" 32 . Die Anklänge an ähnliche Diagnosen, die Adorno oder Hannah Arendt wenig später treffen werden und die an ihrer Aktualität für die heutige „post-truth“-Ära nichts verloren haben, lassen sich kaum überhören ${ }^{33}$.

31. — Vgl. J. v. Moltke, The Curious Humanist..., a.a.O., S. 44. Allerdings verwendet Kracauer hier anders als im Deutschen den Begriff ,soul“/Seele, der auch für die Arbeit an From Caligari to Hitler zentral werden wird: die NS-Propaganda ziele darauf ab, „to make [the audience's] souls work in the interest of Nazi Germany. They treated souls like prisoners of war [and] endeavored to duplicate in the field of psychology Germany's achievements in Europe.“ Siegfried Kracauer, „Propaganda and the Nazi War Film", in: Ders., From Caligari to Hitler: A Psychological History of the German Film, Princeton, Princeton University Press, 2004, S. 273-307, hier S. 288.

32. - Ebd.

33. - „Its [propaganda's - J.v.M.] ultimate aim is probably not so much the selling of a false argumentation as, indeed, the complete breakdown of a logical sense within the listeners and eventually the collapse of any meaning that the idea of truth may have for them“ (T.W. Adorno, The Psychological Techniques..., a.a.O., S. 36); „The result of a consistent and total substitution of lies for factual truth is not that the lies will now be accepted as truth, and the truth be defamed as lies, but that the sense by which we take our bearings in the real world - and the category of truth vs. falsehood is among the 
„Reality was put to work faking itself“, schreibt Kracauer und beschreibt Triumph des Willens durchaus treffend als eine „Transfiguration der Wirklichkeit" (transfiguration of reality), wie sie für das ,ganze totalitäre System“ charakteristisch sei. Nur wenige Jahre nach seinen ersten Studien zum Thema begreift Kracauer hier die Propaganda ,as a tool to destroy the disturbing independence of reality“. Damit errichte diese wiederum einen, impregnable intellectual ,West Wall' against the dangerous invasion of democratic ideas" - womit klar wird, dass Kracauer hier „Wirklichkeit" und „Demokratie“ unter der Hand in eins setzt ${ }^{34}$. Hinter der Maske des Films darf keine Wirklichkeit hervorscheinen, hinter der Maske der Propaganda keine Demokratie.

Das ist für sich genommen nicht unproblematisch, sind doch Ideologie und Propaganda nicht einfach das Andere der Demokratie, sondern dieser inhärent; umgekehrt muss auch die schlimmste Propaganda sich noch auf irgendeine Wirklichkeit beziehen, um aus ihr Funken zu schlagen. Mit anderen Worten: eine ästhetische Analyse darf nicht dazu verleiten, Propaganda und Faschismus mit Illusion und Demokratie mit Wirklichkeit und transparenter Information in eins zu setzen ${ }^{35}$. Begreift man allerdings die Fragestellung im emphatischen Sinne als ästhetische, wie Kracauer das durchweg nahelegt, dann indiziert der Wirklichkeitsbegriff nicht bloß ein politisches, sondern auch ein stilistisches Paradigma - nämlich dasjenige des Realismus.

Ich habe an anderer Stelle nachzuweisen versucht, wie wichtig die Kontinuität der gedanklichen Entwicklung ist, die Kracauer im amerikanischen Exil von den ersten Propagandaarbeiten über From Caligari to Hitler bis zur Theory of Film durchmacht ${ }^{36}$. Diese Kontinuität lässt sich nun mit Blick auf Benjamin noch einmal präzisieren als chiasmatische Umkehrung. Hatte Benjamin der Gefahr der Ästhetisierung der Politik - oder mit Kracauer gesprochen: der Transfiguration der Wirklichkeit durch Propaganda - durch die Politisierung der Ästhetik begegnen wollen, so geht es Kracauer in genauer Entsprechung um die Rückbesinnung des Films auf das, was Propagandaästhetik eskamotiert: um ein Sich-Einlassen mit der Realität. Dafür prägt er den Begriff der Errettung der äußeren Wirklichkeit (redemption of physical reality),

mental means to this end - is being destroyed“ (Hannah Arendt, „Truth and Politics“, in: Dies., Between Past and Future. Eight Exercises in Political Thought, New York, Penguin, 1993, S. 257). Vgl. Lee McIntyre, Post-Truth, Cambridge, MIT Press, 2018.

34. - S. Kracauer, „Propaganda and the Nazi War Film“, a.a.O., S. 290.

35. - Vgl. hierzu die Debatte zwischen Kracauer und Meyer Schapiro, in welcher dieser jenem genau diese unterlassene Unterscheidung vorwirft, und die Ingrid Belke anhand des Briefwechsels nachzeichnet in ,,Das Geheimnis des Faschismus liegt in der Weimarer Republik': Der Kunsthistoriker Meyer Schapiro über Kracauers erstes Filmbuch“, Filmexil H.4, 1994, S. 35-49.

36. — J. v. Moltke, The Curious Humanist..., a.a.O. 
den wir seit Theory of Film als Kern von Kracauers filmtheoretischem Realismus begreifen, und der sich nun als ferne Antwort auf Benjamins politisches Manifest im Nachwort zum Kunstwerkaufsatz verstehen lässt ${ }^{37}$. Hatte Benjamin hier gefordert, die faschistische Ästhetisierung der Politik durch die Politisierung der Kunst zu bekämpfen, so argumentiert Kracauer nun, weit weniger polemisch und auf Umwegen von der Propagandaarbeit zur Filmtheorie, dass auf die faschistische Propaganda eine zutiefst realistische Kunst(form) - der Film - zu antworten habe, welche die propagandistisch verformte Wirklichkeit wieder in ihr Recht einsetze: statt der propagandistischen Transfiguration des Realen also filmischer Realismus als zentrale theoretische Figur.

Kritiker haben wiederholt die Entpolitisierung Kracauers im amerikanischen Exil beklagt. Liest man Theory of Film jedoch von den Propagandaarbeiten her und behält dabei das intertextuelle Gespräch mit Benjamin im Ohr, so hallt der Chiasmus von Ästhetik und Politik selbst in der quasi-religiösen Rede von der Errettung bzw. Erlösung der Wirklichkeit im Film noch nach: Das Politische ist dort im dialektischen Sinne aufgehoben.

$\begin{array}{rrl}\begin{array}{r}\text { Ästhetisierung der } \\ \text { Politik }\end{array} & \begin{array}{l}\text { Politisierung der } \\ \text { Ästhetik } \\ \text { Faschismus } \\ \text { Ästhetik }\end{array} & \begin{array}{l}\text { Kommunismus } \\ \text { Politik }\end{array} \\ \text { Propaganda } & & \text { Wirklichkeit } \\ \text { Transfiguration der } & \text { Kracauer } & \text { Realismus } \\ \text { Wirklichkeit } & & \begin{array}{l}\text { Errettung der } \\ \text { Wirklichkeit }\end{array}\end{array}$

Abbildung 1: Chiasmus bei Benjamin und Kracauer (C J. von Moltke)

\section{Coda: „Cultural Aspects of National Socialism“ und die Dialektik der Aufklärung}

Horkheimer hatte in den Verhandlungen mit Kracauer über den Propagandatext schon früh angekündigt, das Institut könne den Aufsatz, wenn er sich zur Publikation nicht eignen sollte, ,dann eben, wie manche

37. - In diesem Zusammenhang ist es hoch relevant, dass Kracauer in seinen Vorüberlegungen zu einer Theorie des Films über die Totenmaske nachdenkt, die hinter jedem Filmbild lauert - eine Formulierung, mit der auch der Propagandatext endet. 
andere wichtige Arbeit, unserem Archiv ein[ordnen]"38. Wenige Jahre später setzte nicht nur Kracauer selbst nach seiner Übersiedlung in die USA diese Arbeit in seinen Propagandastudien für das MoMA fort, die, wie oben skizziert, schließlich in From Caligari to Hitler mündeten (und dort selbst noch als Anhang auftauchen); auch Adorno und Horkheimer sollten Kracauers Arbeit noch einmal aus dem Archiv hervorholen, in welches sie diese eingeordnet hatten. So spielt das gescheiterte Projekt des Propagandaaufsatzes in der Projektskizze für eine umfangreiche, mehrbändige Studie des Nationalsozialismus eine Rolle, welche das Institut als Förderungsantrag bei der Rockefeller Foundation einreichte. Diese Studie trug zunächst den Titel ,Studies in German Culture and Society“, später „Cultural Aspects of National Socialism“39. Das Institut veranschlagte dieses Projekt außerordentlich hoch: es sollte Inhalte und Methoden bündeln, an denen die Arbeit der Gruppe in ihrer Kontinuität auch im neuen amerikanischen Umfeld lesbar werden sollte ${ }^{40}$. Ganz im Sinne von Kracauers Absicht, das ,aus dem alten Unterbau unableitbare" Phänomen des Nationalsozialismus auf der Ebene der Propaganda zu verorten, wollte das Institut für Sozialforschung mit diesem groß angelegten Projekt ,die Kräfte analysieren, die drohen, das Bewußtsein eines demokratischen Volkes in sein Gegenteil zu pervertieren“"41. Zu diesen bewusstseinsverändernden Kräften rechneten Adorno und Horkheimer in den frühen Projektentwürfen auch die Propaganda. In diesem Zusammenhang bot Adorno im Sommer 1940 an, für den Antrag ,den Entwurf des Propagandaabschnitts [...] im Anschluß an den Kracaueraufsatz, der uns doch einmal zu etwas gut sein soll, selber aus[zu]arbeiten" ${ }^{42}$. (So sieht es aus, wenn das Institut die Arbeiten anderer ,seinem Archiv einordnet“...). Für die endgültige Fassung des Antrags, welcher letztendlich von der Rockefeller Foundation abgelehnt wurde, ersetzte das Institut zwar die Propagandaanalyse durch

38. - Brief Horkheimer an Kracauer, zit. in: Totalitäre Propaganda, a.a.O., S. 839.

39. - Vgl. hierzu Roderick Stackelberg, ,„Cultural Aspects of National Socialism“: An Unfinished Project of the Frankfurt School“, Dialectical Anthropology, H.12, 1988, S. 253-260.

40. — ,The studies [...] have grown out of the intellectual interests and the actual experiences of the members over a period of years. In a sense they will contain the results of the thinking about Germany and the modern world which the members of the group have reached up to this time." Max Horkheimer, Entwurf des Anschreibens an die Rockefeller Foundation. Na 1 Nachlass Max Horkheimer 696 - Cultural Aspects of National Socialism. A Research Project (1941). http://sammlungen.ub.uni-frankfurt.de/ horkheimer/content/pageview/6741198 (Zugriff am 23.03.2020). ,[T] sents in microcosm the interdisciplinary collaborative model typical of the Institute's work in the 1930s.“ R. Stackelberg, „,Cultural Aspects...““, a.a.O., S. 253.

41. - Horkheimer Nachlass, Herv. im Original. http://sammlungen. ub.unifrankfurt.de/horkheimer/content/pageview/6741113 (Zugriff am 23.03.2020).

42. - T.W. Adorno, Briefwechsel 4.2, a.a.O., S. $76 f$. 
einen breiteren kulturkritischen Ansatz ${ }^{43}$. Doch zeigt sich an dieser letztlich kleinen Episode die Vernetzung der Forschungsansätze, in welcher auch Kracauers Propagandaarbeit zu verorten ist. Denn so wie dessen Thesen für den Antrag noch einmal relevant geworden waren, so floss die Arbeit am Antrag in den Folgejahren in alle wesentlichen Arbeiten der Institutsmitglieder mit ein, von den mehrbändigen Studies in Prejudice über Horkheimers Eclipse of Reason bis hin zur Dialektik der Aufklärung. Umgekehrt führt aber der Bogen von dieser auch zurück auf Kracauers Argumentation in der Propagandaarbeit, wo er das dialektische Grundprinzip festgehalten hatte, das ,jeder neue technische Fortschritt auch das Risiko eines neuen humanen Rückschritts herauf[beschwört]“". Diesen Satz formuliert Kracauer nicht zufällig mit Bezug auf ein vorangegangenes Goebbels-Zitat zum Fortschritt der technischen Medien vom Radio zum Fernsehen, welchen die Dialektik der Aufklärung im Kulturindustrie-Kapitel analysieren wird. Hier verschränken sich Aufklärung und Massenbetrug sowohl im technologischen Fortschritt medialer Kultur als auch in der Figur der Reklame, deren Nähe zur Propaganda Adorno schon in den Vorgesprächen zu Kracauers Aufsatz reklamiert und die dieser auch in seinen Skizzen herausgestrichen hatte. Im überlieferten Manuskript heißt es dann bei Kracauer resümierend, wenn auch vorsichtig: ,es scheint [...] als eröffneten die Siege der Menschheit über die Natur dieser die Gelegenheit ungeahnter Siege über die Menschheit; als sei das Wachstum der Glückschancen unweigerlich an das des Verhängnisses gebunden“ (59) eine Einsicht, die ebenso wie Formulierungen im „Ornament der Masse“ oder im Photographie-Aufsatz von 1927 die zentrale These der Dialektik der Aufklärung vorausnimmt.

Mit solchen Einsichten haben wir allerdings den Boden der nationalsozialistischen Propaganda als solcher verlassen und das Feld der modernen Medienlandschaft auch in demokratischen Gesellschaften betreten. Das war auch die dedizierte Absicht des "German Project“" gewesen, das die generativen Momente des Faschismus schon in der

43. - In einem Entwurf von Adorno, der vermutlich auf Anfang $1941 \mathrm{zu}$ datieren ist, heißt es: „Der Glaube, daß Hitler die Massen durch eine überlegene Propagandatechnik im Gegensatz zu ihren eignen Interessen, verführt' habe, scheint uns naiv." Horkheimer Nachlass, http://sammlungen.ub.uni-frankfurt.de/horkheimer/ content/pageview/6741114_(Zugriff am 23.03.2020).

Stackelberg weist nach, dass die ersten Entwürfe die Wurzeln des Faschismus in die Weimarer Demokratie zurück verfolgen, wenn nicht gar im Liberalismus als solchem (und mithin potenziell auch in den USA) nachweisen wollten; der fertige Antrag in seiner „final, diluted Version“ nimmt die Radikalität dieser Hypothesen zurück und widmet sich stattdessen der spezifischen Verfasstheit der nationalsozialistischen Kultur. Stackelberg hält zurecht fest, dass die ursprünglichen Hypothesen schließlich Eingang in andere Arbeiten der Institutsmitglieder finden - allen voran in die Dialektik der Aufklärung. 
Kultur der Weimarer Republik hätte suchen wollen, so wie Kracauer es dann einige Jahre später am Weimarer Kino beispielhaft durchführte. Und obgleich sich die theoretische und methodologische Linie des Instituts für Sozialforschung ebenso wie Kracauer in verschiedener Hinsicht den Dichotomien des Kalten Krieges fügten, sollte der hier formulierte dialektische Ansatz, der schon in der Demokratie den Keim des Totalitären sah, in vielen Arbeiten wirksam bleiben - sei es in den Minima Moralia oder eben in der Dialektik der Aufklärung, welcher Kracauer sogar noch mit der Theory of Film näher ist als es scheint. Jenseits der hier nur teilweise nachgezeichneten philologischen Spuren allerdings bleibt die unverminderte Aktualität der Propagandastudien aus dem Umfeld des Instituts für Sozialforschung festzuhalten - ob diese nun seinerzeit die Zustimmung der Mitglieder fanden oder dem Narzissmus der kleinen Differenzen zum Opfer fielen und, wie Kracauers Propagandaarbeit, nie gedruckt wurden. Denn Texte wie Totalitäre Propaganda und der Kunstwerkaufsatz, die Dialektik der Aufklärung und die Studies in Prejudice, Löwenthals Prophets of Deceit und Adornos Psychological Techniques - sie verweisen gemeinsam auf einen Zusammenhang von Demagogie und medial forcierter Verunsicherung, der noch heute und auch in scheinbar demokratisch verfassten Gemeinwesen wirksam ist. Zurecht betrachtete Adorno „das Nachleben des Nationalsozialismus in der Demokratie als potenziell bedrohlicher denn das Nachleben faschistischer Tendenzen gegen die Demokratie" ${ }^{44}$. Angesichts populistischer Machtverschiebungen, die den Niedergang kritischer Rationalität mit der Aufwertung von Verschwörungstheorien forcieren, die unliebsamen Fakten mit der Berufung auf ,alternative facts“ oder auf die faktische Macht des Gefühls begegnen, und deren kritische Öffentlichkeit von „Alternative Influencer Networks“ in sozialen Medien abgelöst wird, lohnt die Relektüre solcher Arbeiten zur totalitären Propaganda ebenso wie die Rückbesinnung auf Kracauers damals unveröffentlicht gebliebenen Text.

44. - Theodor W. Adorno, „Was bedeutet: Aufarbeitung der Vergangenheit“, in: Ders., Kulturkritik und Gesellschaft Bd.2, Frankfurt a.M., Suhrkamp, 2003, S. $555 f$. 
